\title{
Effects of inoculating fungi on agilawood formation in Aquilaria sinensis
}

\author{
CUI JinLong ${ }^{1,2}$, GUO ShunXing ${ }^{1 *}$, FU ShaoBin ${ }^{3}$, XIAO PeiGen $^{1} \&$ WANG MengLiang ${ }^{2}$ \\ ${ }^{1}$ Institute of Medicinal Plant Development, Chinese Academy of Medical Sciences \& Peking Union Medical College, Beijing 100193, China; \\ ${ }^{2}$ Institute of Applied Chemistry, Shanxi University, Taiyuan 030006, China; \\ ${ }^{3}$ Pharmacy School, Zunyi Medical College, Zunyi 563003, China
}

Received December 5, 2012; accepted January 28, 2013; published online May 31, 2013

\begin{abstract}
Agilawood is a costly heartwood medicine obtained from Aquilaria sinensis with active ingredients mainly composed of volatile and semi-volatile substances. However, the formation time of agilawood is quite long and little is known about its formation mechanism. Two highly active fungi obtained from natural agilawood were inoculated on A. sinensis trees to understand their interaction processes and elucidate the transformation rules of induced chemical compositions within different test periods. The results demonstrated that the fungi could successfully colonize living tissues and cells and activate the host defense system, resulting in agilawood accumulation. With increasing time, the main components of $A$. sinensis converted into constituents or analogs of agilawood and the host exhibited "self-injury" to prevent fungal intrusion and protect other tissues. The data presented here could provide scientific basis for producing agilawood with the two new fungi in a safe, feasible, and sustainable manner without destroying rare Aquilaria plants.
\end{abstract}

agilawood, Aquilaria sinensis, fungus, mutual relationship, GC-MS analysis

Citation: $\quad$ Cui J L, Guo S X, Fu S B, et al. Effects of inoculating fungi on agilawood formation in Aquilaria sinensis. Chin Sci Bull, 2013, 58: 3280-3287, doi: $10.1007 / \mathrm{s} 11434-013-5856-5$

In China, agilawood, also known as "chenxiang," is a resinembedded heartwood that forms in Aquilaria sinensis (Lour.) Gilg. (Thymelaeaceae), the only Aquilaria species distributed in Hainan, Yunnan, Guangxi, and Guangdong [1,2]. Only $1 \%-2 \%$ of the trees in natural forests contain agilawood even after 30 years of growth. Agilawood generates only in cut or wounded tissues and cannot form in normal tissues of A. sinensis. The immune system of the host is believed to be activated after infection by some fungi or insects $[3,4]$. During agilawood formation, the main components of $A$. sinensis gradually transform into the major components of agilawood, such as chromones, sesquiterpenes, and other aromatic compounds, and the pale-colored and light wood transforms into a dark and dense aromatic resin-embedded heartwood [4].

Several fungi with induction activity, such as Menanotus

*Corresponding author (email: sxguo2006@yahoo.com.cn) flavolives, have been obtained from agilawood [5]. However, the formation mechanism of agilawood remains unclear. No published report of the successful application of fungi in agilawood production yet exists because the induction activity of available fungi is not adequately high, thereby severely limiting drug supplies and causing prices to increase to up to US $\$ 100000 / \mathrm{kg}$ for superior, pure material. Thus, systematic research on the interaction mechanism between fungi and their host and demonstrations of the transformation rule of chemical composition in induced heartwood are highly necessary. Our previous work indicated that agilawood contains an abundance of fungi and that fungal elicitation with high activity induces fragrant ingredient production [6,7]. In the present study, two fungi, YNAS06 and YNAS08, were inoculated on A. sinensis to explore the interaction process between fungi and host and investigate the chemical composition of induced agilawood at 2, 4, 6, and 8 months. This work is expected to provide scientific basis for 
the application of the proposed induction method.

\section{Materials and methods}

\subsection{Solid culture of test fungi}

The test fungi, YNAS06 (GenBank No. GU355650) and YNAS08 (GenBank No. GU355652), which are Xylaria sp. and Lasiodiplodia sp., respectively [6], were isolated from natural agilawood in Yunnan Province, China, in March 2009. The test fungi were cultured with solid medium [7] at $25^{\circ} \mathrm{C}$ for $20 \mathrm{~d}$ before inoculation on trees. High-quality hyphae with solid culture medium were used as inoculation materials to inoculate $A$. sinensis trees.

\subsection{Inoculation of fungal materials}

Holes with a surface size of $1 \mathrm{~cm}$ in diameter were punched into the trunk of 10-year-old healthy trees distributed in Chengmai County, Hainan Province, China, and identified by Prof. Guo Shunxing. The depth of the holes was $3 \mathrm{~cm}$ and the spacing between holes was $15 \mathrm{~cm}$. Then, $2 \mathrm{~g}$ of solid fungal material was quickly inoculated into the hole, covered with sterile cotton, and fixed with fiber. Each tested group included four holes in the same tree: the blank control (Bc, no filling), the negative control ( $\mathrm{Nc}$, solid medium filling but without fungi), YNAS06, and YNAS08. Tests for each group were repeated thrice on different trees.

\subsection{Collection of induced agilawood}

The induced agilawood was collected when the fungus inoculated on the plant stems of A. sinensis had grown for 2, 4, 6 , and 8 months. The inoculation site changed in color from white to brown and was dug up by a chisel. Fresh samples for morphological observation were promptly fixed with fixing solution. Samples for chemical analysis were stripped of their surrounding bark and white xylem, cut into small pieces, dried in the shade, and then set aside.

\subsection{Morphological characterization}

Morphological observations of the interactions between the fungi and their host were performed using a light microscope (Axio Imager AI, ZEISS) and scanning electron microscope (SEM, JSM-65510LV, JEOL). The materials were fixed with formalin-acetic acid [ethanol (70\%): acetic acid: formalin $(37 \%$ to $40 \%$ formaldehyde $)=90: 4.5: 5.5(\mathrm{v} / \mathrm{v} / \mathrm{v})]$. A week later, the fixed materials were softened with softener [ethanol (50\%): glycerol $=1: 1(\mathrm{v} / \mathrm{v})$ ] at $95^{\circ} \mathrm{C}$ for $10 \mathrm{~h}$. The procedure for sample treatment was an improved method described by Oriani and Scatena [8]. The SEM was used to observe sample ultrastructures and verify results under a light microscope. The sheared materials used for SEM were fixed in $2.5 \%$ phosphoric acid glutaraldehyde $\left(0.2 \mathrm{~mol} \mathrm{~L}^{-1}\right.$ phosphate buffer and $25 \%$ glutaraldehyde in $100 \mathrm{~mL}$ of double distilled water). The treatment methods are described in ref. [9].

\subsection{Extraction of volatile and semi-volatile constituents of induced agilawood}

Well-dried samples were powdered and passed through 20 meth sieves. The volatile oils were obtained by hydrodistillation in accordance with the method in Pharmacopoeia of the People's Republic of China [1]. The distilled oil was dried over anhydrous sodium sulfate and stored in a freezer at $-20^{\circ} \mathrm{C}$ until analysis.

1.6 Analysis of induced agilawood by gas chromatography-mass spectrometry (GC-MS)

A gas chromatograph coupled with a mass spectrometer (7890A/5975C, Agilent) was used to analyze the composition of volatile materials. The instrument was equipped with a HP-5 capillary column ( $30 \mathrm{~m} \times 0.25 \mathrm{~mm} \times 0.25 \mu \mathrm{m}$, Agilent) for chromatographic separation with nitrogen (purity $>99.999 \%$ ) as the carrier gas at a constant flow rate of $1.0 \mathrm{~mL} \mathrm{~min}{ }^{-1}$. The injector temperature was $250^{\circ} \mathrm{C}$. Oven temperature programming was performed as follows: initial temperature of $80^{\circ} \mathrm{C}$, held for $1 \mathrm{~min}$, raised at a rate of $10^{\circ} \mathrm{C} \mathrm{m^{-1 }}$ to $150^{\circ} \mathrm{C}$, raised to $250^{\circ} \mathrm{C}$ at a rate of $5^{\circ} \mathrm{C} \mathrm{min}^{-1}$, and then held for $2.5 \mathrm{~min}$. The sample $(3 \mu \mathrm{L})$ was injected in pulsed splitless mode and the total analysis time for a GC run was 32 min. The MS was operated in electron impact ionization mode $(70 \mathrm{eV})$. The mass ranged from $\mathrm{m} / \mathrm{z} 40 \mathrm{amu}$ to $\mathrm{m} / \mathrm{z}$ $500 \mathrm{amu}$.

\subsection{Confirmation of inoculation fungus}

Fungi inocula were re-isolated from induced agilawood to verify the authenticity of the induced test. The identification methods were based on the internal transcribed spacer of ribosomal DNA (ITS-rDNA) sequences and the morphological characteristics of the fungus [6]. A pair of primers ITS1 (5'-TCCGTAGGTGAACCTGCGG-3') and ITS4 (5'TCCTCCGCTTATTGATATGC-3') were used for ITS-rDNA amplification. The corresponding ITS-rDNA sequence of re-isolated fungus was then employed for similarity analysis using the BLASTN algorithm against the original inoculation fungus. The test authenticity was determined by the re-isolation rate $(>95 \%)$ of the original inoculation fungus.

\section{Results and discussion}

\subsection{Microscopic characteristics of agilawood formation}

The morphological characteristics of secondary xylem in stem were observed by light microscopy and SEM. The results indicated that the tissues and cells of healthy $A$. 
sinensis xylem were regular and complete under the conditions of no fungal invasion (Figure 1(a)). "Starch grains" in cells were clearly seen (Figure 1(b)). With increasing fungal invasion, the cell walls underwent secondary thickening and
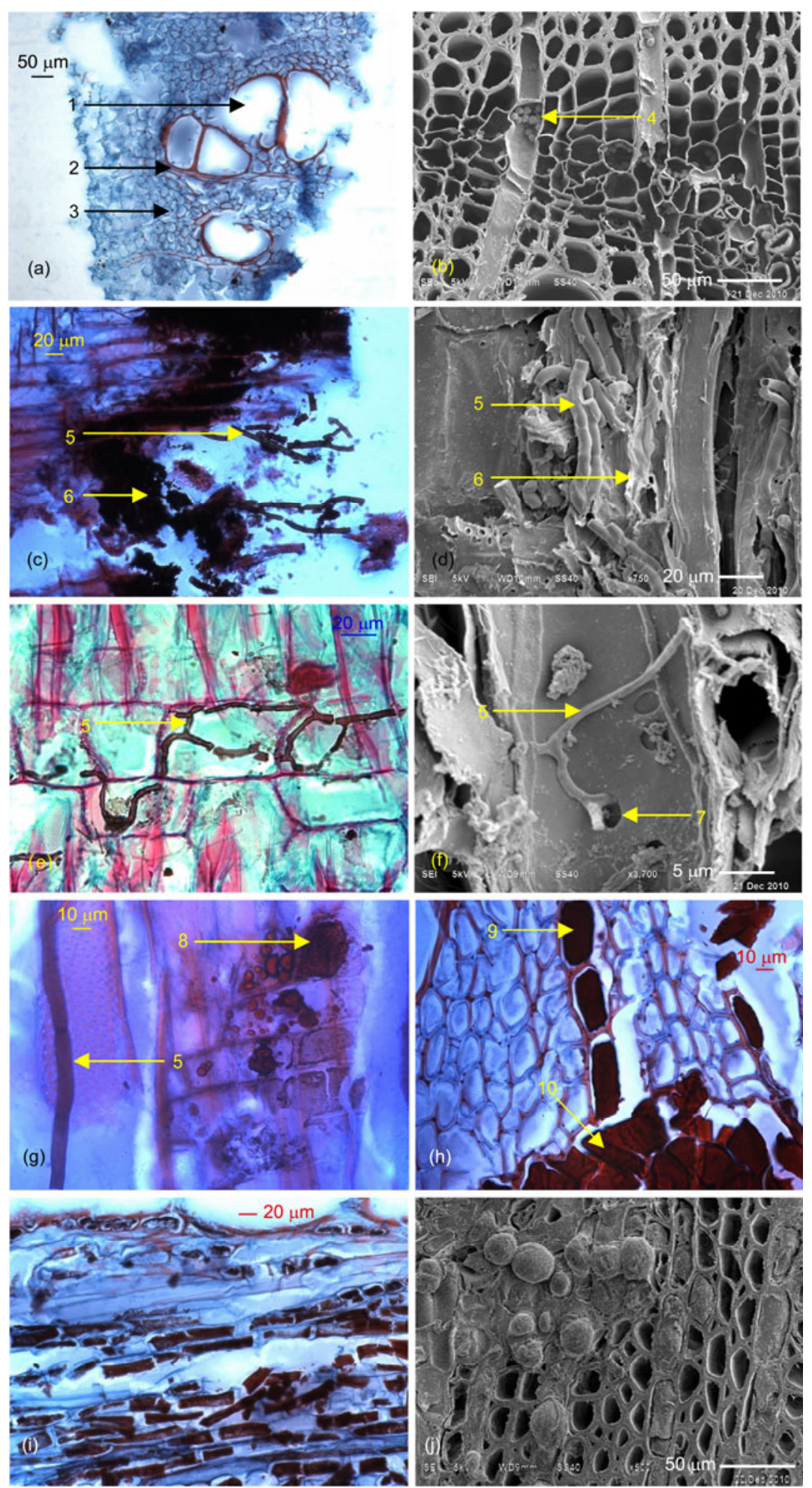

Figure 1 Morphological observations of the interaction between the fungus and its host based on light microscopy and SEM. (a, b) The tissues and cells of healthy A. sinensis xylem as observed by light microscopy and SEM; (c) hyphae were digested; (d) secretions and fungus coexisted and fused together in the inoculation site; (e) reproduction of hyphae from one cell to the other; (f) paired pits are the passageways of hyphae growing to other cells; (g) secretions were produced during fungal invasion; (h) blocked transfusion tissues; (i, j) the microstructure of induced agilawood was similar to natural agilawood. 1, vessel cells; 2 , ray cells; 3 , fiber cells; 4, starch grain; 5, hypha; 6, coexistence of hyphae and secretions; 7, cell pits; 8, secretions; 9, blocked ray tissue; 10, blocked vessel. 
lignification. In these positions, some secretions were produced and coexisted with fungus. The "starch grains" were observed to disappear and some hyphae tended to be digested (Figures 1(c) and (d)). Some inoculated fungi were observed to multiply in cells and continued to grow and invade new healthy tissues. These fungi mostly extended along the axes of cells and entered adjacent cells through cell pits (Figures 1(e) and 1(f)). The "defense response" of the host was triggered after fungal invasion (Figure 1(g)). Significant amounts of secretion were produced, blocking transfusion tissues, such as vessel cells and ray cells, and cutting off the nutritional supply of the fungus (Figure 1(h)). Secretions adhered to the fungus decreased fungal growth. Thus, the growth of hyphae in tissues first occurred rapidly and then slowly according to observations of the materials at different test times. The results showed that the fungi invading tissues gradually lignified and formed structures similar to natural agilawood (Figures 1(i) and 1(j)). A significant amount of secretion was produced in neighboring tissues (no fungi invasion) of fungal-invasion sites, where transfusion tissues were also blocked and formed a "thylose" structure, which is a type of agilawood structure. Farther away from this position, the tissues and cells were arranged orderly, similar to the arrangement of healthy A. sinensis. This phenomenon demonstrates that the host prevents the transfusion tissue from resisting fungal invasion to protect the other healthy tissues. Thus, the problem of fungal infection was solved by increasing the possibility of survival. We refer to this phenomenon as "self-injury", which is a new discovery.

Agilawood is known to form from A. sinensis because $A$. sinensis plants respond to foreign body attacks with defense responses [10,11]. Morphological observations in the present study agree with this perspective. When fungi invade tissues, the host not only retards fungal growth but also interrupts its own nutritional supply by plugging conduction tissue. This behavior is described as "self-injury" to protect other tissues. Self-injury allows the host to maintain normal growth even during foreign invasion.

\subsection{The chemical composition analysis of induced agilawood at 2, 4, 6 and 8 months}

The major components of the volatile oil from healthy $A$. sinensis and natural agilawood were identified. As shown in Table 1, eight components were identified in healthy $A$. sinensis wood, representing $93.489 \%$ of the total volatile materials. Dominant constituents included dibutyl phthalate (DP), phenol,2,2'-methylenebis[6-(1,1-dimethylethyl)-4-methyl] (PMDM), and hydrocarbons. Nine components were identified in natural agilawood, representing $88.612 \%$ of the total volatile materials. Except for DP (35.689\%) and PMDM $(13.259 \%)$, other substances such as spirovetiva-3,7(11)-dien12-al, 4-phenyl-2-butanone (1.608\%), beta-vatirenene1,8adimethyl-7-propan-2-ylidene-1,2,6,8-tetrahydron (9.381\%), baimuxinal $(2.423 \%)$, and $\alpha$-humulene $(2.514 \%)$ were seen as characteristic components of the agilawood samples.

Tables 2 and 3 show the main compositions of the test

Table 1 Main volatile components of the oil from healthy A. sinensis wood and agilawood samples collected from Hainan Province ${ }^{\text {a) }}$

\begin{tabular}{|c|c|c|c|c|c|c|}
\hline \multirow[b]{2}{*}{ No. } & \multirow[b]{2}{*}{ Compounds } & \multirow[b]{2}{*}{ Formula } & \multirow[b]{2}{*}{$\mathrm{RI}^{*}$} & \multirow[b]{2}{*}{ Identification } & \multicolumn{2}{|c|}{ Relative content $(\%)^{* *}$} \\
\hline & & & & & A. sinensis & Agilawood \\
\hline 1 & Pentadecane & $\mathrm{C}_{15} \mathrm{H}_{32}$ & 1498 & $\mathrm{RI}^{[12]} / \mathrm{MS}$ & $0.512 \pm 0.012$ & $--^{* * *}$ \\
\hline 2 & Dibutyl phthalate & $\mathrm{C}_{16} \mathrm{H}_{22} \mathrm{O}_{4}$ & 1962 & $\mathrm{RI}^{[12]} / \mathrm{MS}$ & $63.983 \pm 2.643$ & $35.689 \pm 0.953$ \\
\hline 3 & Eicosane & $\mathrm{C}_{20} \mathrm{H}_{42}$ & 1999 & $\mathrm{RI}^{[13]} / \mathrm{MS}$ & $0.353 \pm 0.039$ & - \\
\hline 4 & E-7-Octadecene & $\mathrm{C}_{18} \mathrm{H}_{36}$ & 1640 & $\mathrm{RI}^{[12]} / \mathrm{MS}$ & $1.318 \pm 0.163$ & - \\
\hline 5 & E-5-Octadecene & $\mathrm{C}_{18} \mathrm{H}_{36}$ & 1775 & $\mathrm{RI}^{[13]} / \mathrm{MS}$ & $0.653 \pm 0.121$ & - \\
\hline 6 & Cyclotetradecane & $\mathrm{C}_{14} \mathrm{H}_{28}$ & 1673 & $\mathrm{RI}^{[13]} / \mathrm{MS}$ & $0.985 \pm 0.136$ & - \\
\hline 7 & Tetracosane & $\mathrm{C}_{30} \mathrm{H}_{62}$ & 2400 & $\mathrm{RI}^{[13]} / \mathrm{MS}$ & $0.496 \pm 0.005$ & - \\
\hline 8 & Phenol,2,2'-methylenebis[6-(1,1-dimeth-ylet hyl)-4-methyl- & $\mathrm{C}_{23} \mathrm{H}_{32} \mathrm{O}_{2}$ & 2398 & $\mathrm{RI}^{[13]} / \mathrm{MS}$ & $25.189 \pm 0.622$ & $13.259 \pm 0.599$ \\
\hline 9 & Spirovetiva-3,7(11)-dien-12-al & $\mathrm{C}_{15} \mathrm{H}_{22} \mathrm{O}$ & 1805 & $\mathrm{RI}^{[13]} / \mathrm{MS}$ & - & $2.794 \pm 0.047$ \\
\hline 10 & $\begin{array}{l}\text { 2(1H)Naphthalenone,3,5,6,7,8,8a-hexahydro-4,8a-dimethyl-6-(1-methyle } \\
\text { thenyl)- }\end{array}$ & $\mathrm{C}_{15} \mathrm{H}_{22} \mathrm{O}$ & 1772 & $\mathrm{RI}^{[13]} / \mathrm{MS}$ & - & $8.747 \pm 0.126$ \\
\hline 11 & $\begin{array}{l}\text { (1R,3R)-3-isopropenyl-2,2-dimethylcyclobutylmethyl } \\
\text { 3-methyl-3-butenoate }\end{array}$ & $\mathrm{C}_{15} \mathrm{H}_{24} \mathrm{O}_{2}$ & 1526 & $\mathrm{RI}^{[13]} / \mathrm{MS}$ & - & $10.765 \pm 0.233$ \\
\hline 12 & 6-isopropenyl-4,8a-dimethyl-1,2,3,5,6,7,8,8a-octahydro-naphthalen-2-ol & $\mathrm{C}_{15} \mathrm{H}_{24} \mathrm{O}$ & 1714 & $\mathrm{RI}^{[13]} / \mathrm{MS}$ & - & $1.432 \pm 0.055$ \\
\hline 13 & 4-phenyl-2-butanone & $\mathrm{C}_{10} \mathrm{H}_{12} \mathrm{O}$ & 1210 & $\mathrm{RI}^{[14]} / \mathrm{MS}$ & - & $1.608 \pm 0.015$ \\
\hline 14 & .Beta.-Vatirenene1,8a-dimethyl-7-propan-2-ylidene-1,2,6,8-tetrahydron & $\mathrm{C}_{15} \mathrm{H}_{22}$ & 1489 & $\mathrm{RI}^{[15]} / \mathrm{MS}$ & - & $9.381 \pm 0.041$ \\
\hline 15 & Baimuxinal & $\mathrm{C}_{15} \mathrm{H}_{24} \mathrm{O}_{2}$ & 1707 & $\mathrm{RI}^{[13]} / \mathrm{MS}$ & - & $2.423 \pm 0.042$ \\
\hline 16 & $\alpha$-Humulene & $\mathrm{C}_{15} \mathrm{H}_{24} \mathrm{O}$ & 1464 & $\mathrm{RI}^{[14]} / \mathrm{MS}$ & - & $2.514 \pm 0.035$ \\
\hline
\end{tabular}

a) *, Retention index (RI) based on a homologous series of normal alkanes; **, values are given as means \pm SD (n=3); ***, “-” not detected. 


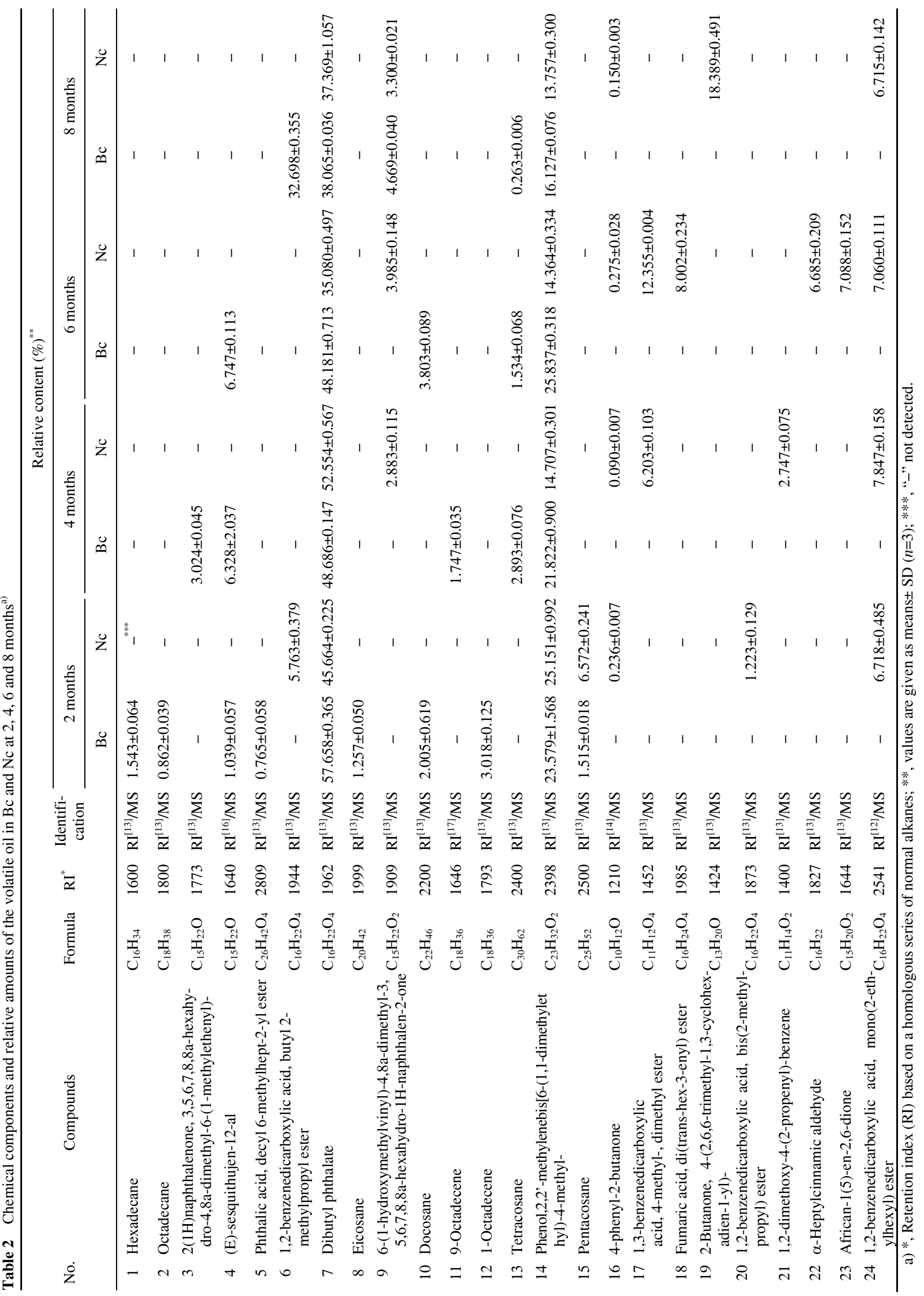




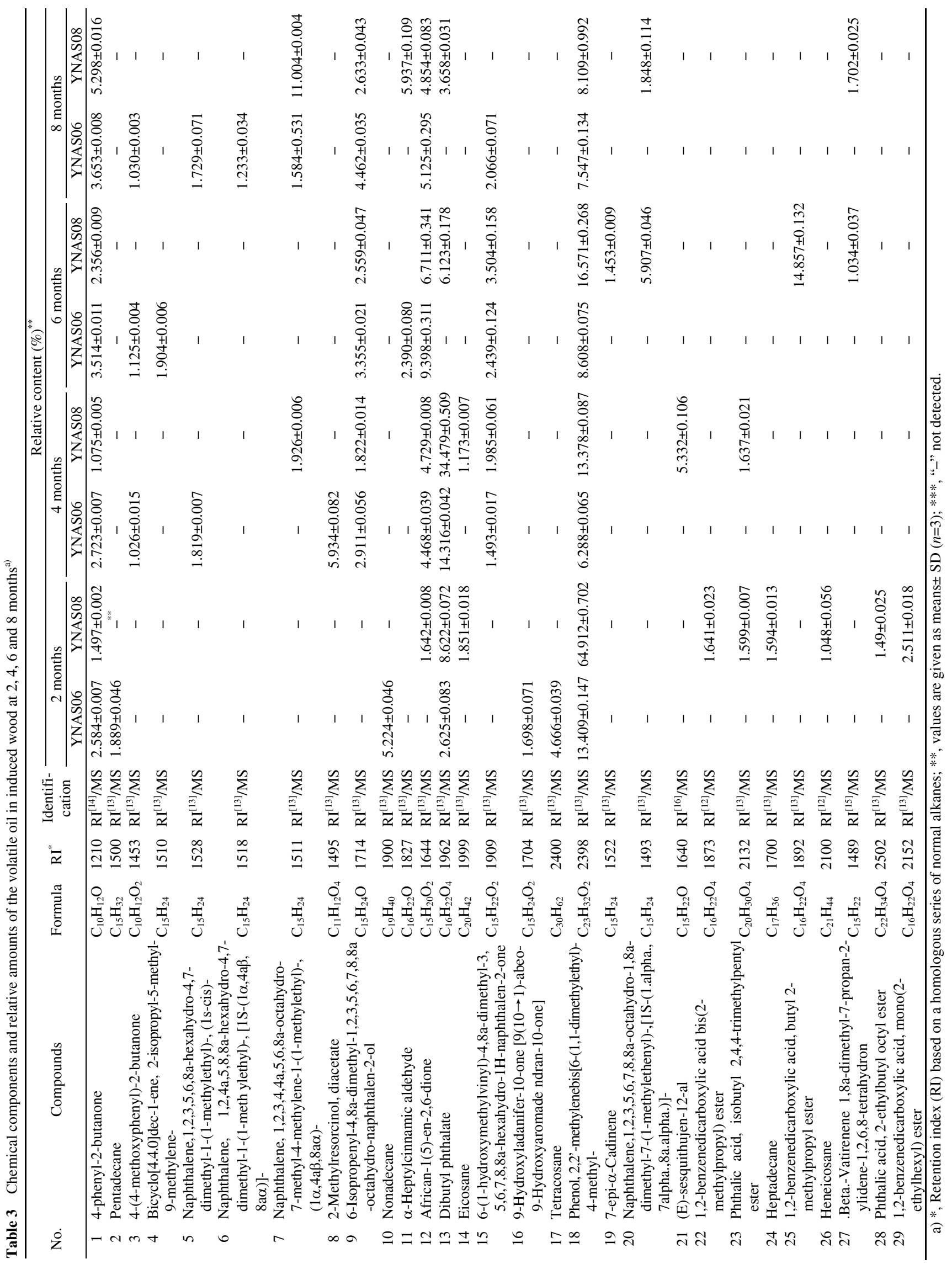


materials, including the blank control, the negative control, the YNAS06-induced agilawood (06IA), and the YNAS08induced agilawood (08IA) at 2, 4, 6, and 8 months. Table 3 shows that the contents of DP and PMDM in YNAS06induced samples decreased from 2 to 6 months. Six months after the induction test, DP disappeared and the PMDM content decreased to within $10 \%$ of the original values $(8.608 \%)$. The content and relative ratio of agilawood analogs increased significantly. Hydrocarbons almost completely disappeared at 8 months. The content and quantity of characteristic compounds or their analogues in agilawood, such as chromones (Table 3, Nos. 1,3) and sesquiterpenoids or their analogues (Table 3, Nos. 5,6,7,9,12,15), increased 8 months after induction. The results showed that the substances in A. sinensis convert to agilawood compounds within 8 months of induction. Table 3 also shows that the GC-MS analysis results of 08IA were similar to those of 06IA. The relative contents of DP and PMDM accounted for $73.534 \%$ of the volatile content of 2-month-old extracts and then gradually decreased to only $11.767 \% 8$ months after induction. The relative content of hydrocarbons, such as eicosane, heptadecane, and heneicosane, was $4.493 \%$ at 2 months and disappeared after 4 months. Sesquiterpenes and their derivatives, (Table 3, Nos. 7,9,12,20,27) accounted for $22.041 \%$ of the volatile materials at 8 months. Table 2 shows that the induced effects and sustainable time significantly differed in the control test. In $\mathrm{Bc}$ and $\mathrm{Nc}$, mechanical damage, which is also regarded as a type of induction method, was observed and some chemicals were converted from A. sinensis. However, with time, reductions in the contents of DP, PMPD, and hydrocarbons decreased. The speed by which aromatic compounds increased in content was not high. Another major type of characteristic ingredient, namely, chromone was not detected.

The results of our GC-MS analysis indicated that the chemical composition of the volatile materials produced rapid transformation, suggesting that fungi promote agilawood formation. During the experiment, fungal invasion first occurred rapidly and then decreased. Plant regions with fungal invasion changed in color from white to brown. These phenomena show that a dynamic process is involved in the interaction between fungi and $A$. sinensis. In this process, the fungi serve as "fungal elicitors" to change the metabolic pathway of the host [18]. Some constituents from fungi work as signal molecules, inducing a series of signal transduction reactions and active gene expression before finally resulting in accumulation of specific secondary metabolites [19]. Thus, the major chemical constituents of $A$. sinensis are converted to sesquiterpenoids, chromones, and aromatic compounds, which are the predominant chemicals in agilawood.

Components such as DP, PMDM, and hydrocarbons were distributed widely in the tissues of A. sinensis trees. These components are poisonous to humans and inedible [20]. The contents of these components decreased in in- duced and natural agilawood because of fungal induction. However, as a traditional medicine used for thousands of years, the lack of a reliable toxicity test deserves further research.

\subsection{Confirmation of inoculation fungus}

According to the re-isolation rate, the planting percentages of YNAS06 and YNAS08 were 98\% and 97\%, respectively, based on their ITS-rDNA sequences and morphological identification. These percentages indicate that the fungi used were advantageous in inducing agilawood and that the test results were credible.

\section{Conclusion}

This work discovered a new agilawood formation, in which the host adopts a "self-injury" mechanism to protect other tissues during fungal invasion. This study also clarified the change rule of induced chemical product by fungal inoculation at the different test times. The theory and practical data in this study will be helpful in improving agilawood production.

This work was supported by the National Natural Science Foundation of China (31170016, 31270383).

1 Chinese Pharmacopeia Commission. Pharmacopoeia of the People's Republic of China. Beijing: Chinese Medical Science and Technology Press, 2010

2 Yang L, Qiao L R, Xie D, et al. z-(z-phenylethyl) chromones from Chinese eaglewood. Phytochemistry, 2012, 76: 92-97

3 Pripdeevech P, Khummueng W, Park S K. Identification of odor-active components of agarwood essential oils from Thailand by solid phase microextraction-GC/MS and GC-O. J Essent Oil Res, 2012, 23: 4653

4 Naef R. The volatile and semi-volatile constituents of agilawood, the infected heartwood of Aquilaria species: A review. Flavour Frag J, 2011, 26: 73-89

5 Qi S Y, Lu B Y, Zhu L F, et al. Formation of oxo-agarospirol in Aquilaria sinensis. Plant Physil Commun, 1992, 28: 336-339

6 Cui J L, Guo S X, Xiao P G. Antitumor and antimicrobial activities of endophytic fungi from medicinal parts of Aquilaria sinensis. J Zhejiang Univ-Sci B, 2011, 12: 385-392

7 Cui J L, Guo S X, Xiao P G, et al. Fungus inducing the formation of agilawood from Aquilaria sinensis. Chin Pharm J, 2012, 47: 335-338

8 Oriani A, Scatena V L. Intracellular papillae of Actinocephalus (Eriocaulaceae-Poales) roots and their interaction with fungi: A light and transmission electron microscopy study. Micron, 2007, 38: 611-617

9 Pathan A K, Bond J, Gaski R E. Sample preparation for SEM of plant surfaces. Micron, 2008, 39: 1049-1061

10 Zhang Z, Yang Y, Wei J H, et al. Advances in studies on mechanism of agilawood formation in Aquilaria sinensis and its hypothesis of agilawood formation induced by defense response. Chin Tradit Herbal Drugs, 2010, 41: 156-159

11 Caillot S, Rat S, Tavernier M L, et al. Native and sulfated oligoglucuronans as elicitors of defence-related responses inducing protection against Botrytis cinerea of Vitis vinifera. Carbohyd Polym, 2012, 87: 1728-1736 
12 Chen H Q, Yang Y, Xue J, et al. Comparison of compositions and antimicrobial activities of essential oils from chemically stimulated agarwood, wild agarwood and healthy Aquilaria sinensis (Lour.) Gilg trees. Molecules, 2011, 16: 4884-4896

13 Tret'yakov K V. Retention Data. NIST Mass Spectrometry Data Center. 2008. Available online: http: //chemdata.nist.gov/mass-spc/pubs/ pittcon-2000/index.htm (accessed on 6 January 2013)

14 Tajuddin S N, Yusoff M M. Chemical composition of volatile oils of Aquilaria malaccensis (Thymelaeaceae) from Malaysia. Nat Prod Commun, 2010, 5: 1965-1968

15 Chou S T, Lai C P, Lin C C, et al. Study of the chemical composition, antioxidant activity and anti-inflammatory activity of essential oil from Vetiveria zizanioides. Food Chem, 2012, 134: 262-268

16 Weyerstahl P, Marschall H, Eckhardt A, et al. Constituents of com- mercial Brazilian Iantana oil. Flavour Fragr J, 1999, 14: 15-28

17 Petersen M A, Poll L, Larsen L M. Comparison of volatiles in raw and boiled potatoes using a mild extraction technique combined with GC odour profiling and GC-MS. Food Chem, 1998, 61: 461-466

18 Fujita M, Fujita Y, Noutoshi Y, et al. Crosstalk between abiotic and biotic stress responses: A current view from the points of convergence in the stress signaling networks. Curr Opin Plant Biol, 2006, 9: $436-442$

19 Zhao J, Davis L C, Verpoorle R. Elicitor signal transduction leading to production of plant secondary metabolites. Biotechnol Adv, 2005, 2: $283-333$

20 Clara M, Windhofer G, Hartl W, et al. Occurrence of phthalates in surface runoff, untreated and treated wastewater and fate during wastewater treatment. Chemosphere, 2010, 78: 1078-1084

Open Access This article is distributed under the terms of the Creative Commons Attribution License which permits any use, distribution, and reproduction in any medium, provided the original author(s) and source are credited. 